\title{
Therapieentwicklungen bei chronisch entzündlichen Darmerkrankungen - neue Strategien und neue Substanzen
}

Die Therapie der chronisch entzündlichen Darmerkrankungen ist von mehr oder weniger gezielten Eingriffen in die Immunregulation geprägt. Folgen einer systemisch wirkenden anti-entzündlichen Therapie sind allerdings mitunter erhebliche immunsuppressive Nebenwirkungen. Diese sind jedoch unmittelbar mit dem Wirkmechanismus der meisten Substanzen verbunden. Die entscheidende Innovation vor mehr als zwei Jahrzehnten war die Einführung der anti-TNF-Therapie, mit der erstmals gezielt durch monoklonale Antikörper gegen das Zytokin TNF eine Effizienzerhöhung über die bislang verfügbaren, unspezifischen Systemtherapeutika (Steroide, Azathioprin) erreicht werden konnte. Die erhebliche Effizienzverbesserung ist jedoch mit einer mitunter auch ebenso erheblichen Immunsuppression vergesellschaftet. Trotzdem haben Therapeutika dieser Substanzklasse (Adalimumab, Golimumab, Infliximab) zu einem sehr deutlichen Sprung in der Behandelbarkeit und Langzeitprognose (auch quoad vitam) der Patienten mit Morbus Crohn und Colitis ulcerosa geführt. Mittlerweile erlauben biosimilare Kopien dieser Substanzen eine adäquate, patientenindividuelle Dosierung zu deutlich reduzierten Kosten.

Die nächste therapeutische Intervention war die Entwicklung eines auf das $\alpha 4 \beta 7$-Integrin gerichteten, blockierenden Antikörpers, Vedolizumab, bei dem erstmalig eine anti-entzündliche Wirkung im Darm ohne immunsuppressive Nebenwirkungen ent- stand. Obwohl anfangs aufgrund des benignen Nebenwirkungsprofils als schwach wirksam kategorisiert, konnte eine doppelblinde, prospektiv-randomisierte Vergleichsstudie gegen Adalimumab sowohl den schnellen Wirkungseintritt als auch eine therapeutische Überlegenheit gegen Adalimumab bei Colitis ulcerosa nachweisen. Mittlerweile sind mit Ustekinumab (Therapieziel ist die Blockade des p40-Proteins, das den Zytokinen, IL-12 und IL23, gemeinsam ist) und Tofacitinib (Therapieziel ist die Inhibition des Janus-Kinase [JAK]-Systems [vorwiegend JAK3, JAK1 und JAK2] und damit eine Multi-ZytokinInhibition) noch zwei weitere Therapieklassen dazu gekommen. Leider besteht nach wie vor eine erhebliche Notwendigkeit, die Therapie zu verbessern: zum Einen sind die langfristigen Effizienzraten nicht ausreichend hoch (insbesondere wenn es zum Anspruch einer Krankheitskontrolle kommt, wo Erfolge in niedrigen zweistelligen Prozentsätzen liegen) und zum Anderen ist die Vermeidung schwerer Nebenwirkungen wichtiges Therapieziel geworden.

Die Notwendigkeit, neue Therapien zu entwickeln hat zu einer intensiven Forschungskompetition geführt, aus der drei Innovationswellen entstanden sind. Dieses sind zum einen JAK1-selektive Inhibitoren, die möglicherweise einige der Nebenwirkungen einer Pan-JAK-Inhibition reduzieren, S1P-Rezeptor-Agonisten, die durch höhere Selektivität für das Ansprechen einzelner Subklassen des S1P-Rezeptors Ef- fizienz und Nebenwirkungen der Muttersubstanz Fingolimod verbessern, sowie spezifische IL-23 Inhibitoren. Gerade die letztere Substanzklasse wird mit großer Spannung erwartet, da die Inhibition von IL-23 über den Mechanismus der p19-Inhibition zu einem durchschlagenden Erfolg in der Psoriasistherapie geführt hat. Phase III-Studien laufen noch für die Anwendung bei Morbus Crohn und Colitis ulcerosa, aber bislang versprechen erste Ergebnisse zwar bessere Effizienzen, zumindest in der Therapie des Morbus Crohn, wobei die Größenordnung von den durchgreifenden Erfolgen bei der Psoriasis noch weit entfernt ist.

\section{Interessenkonflikt}

Die Autoren geben an, dass kein Interessenkonflikt besteht.

\section{Autor}

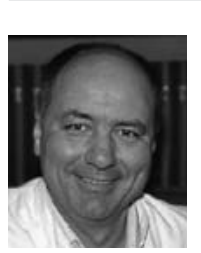

\section{Stefan Schreiber}

Klinik für Innere Medizin I, Universitätslinikum Schleswig-Holstein (UKSH), Christian-Albrechts-Universität zu Kiel, Deutschland

\section{Korrespondenzadresse}

Prof. Dr. med. Stefan Schreiber

Klinik für Innere Medizin I

Universitätsklinikum Schleswig-Holstein

Arnold-Heller-Straße 3

24105 Kiel

Deutschland

s.schreiber@ikmb.uni-kiel.de 